\title{
Butyric acid - a well-known molecule revisited
}

\author{
Katarzyna Borycka-Kiciak ${ }^{1}$, Tomasz Banasiewicz², Grażyna Rydzewska ${ }^{3}$ \\ ${ }^{1}$ Department of General and Gastrointestinal Surgery MCPE, Orlowski Hospital, Warsaw, Poland \\ ${ }^{2}$ Department of General and Endocinological Surgery, and Gastrointestinal Oncology, Poznan University of Medical Sciences, \\ Poznan, Poland \\ ${ }^{3}$ Department of Internal Medicine and Gastroenterology, Division of Inflammatory Bowel Diseases, Central Clinical Hospital \\ of Ministry of Internal Affairs and Administration, Warsaw, Poland
}

Gastroenterology Rev 2017; 12 (2): 83-89

DOI: https://doi.org/10.5114/pg.2017.68342

Key words: butyric acid, irritable bowel syndrome, inflammatory bowel disease, constipation, diarrhea.

Address for correspondence: Katarzyna Borycka-Kiciak MD, PhD, Department of General and Gastrointestinal Surgery MCPE, Orlowski Hospital, 231 Czerniakowska St, 00-461 Warsaw, Poland, phone: +48 502766 961, e-mail: kborycka@interia.pl

\begin{abstract}
The properties of butyric acid, and the role it plays in the gastrointestinal tract, have been known for many years. However, the newest research shows that butyric acid still remains a molecule with a potential that has not as yet been fully exploited. The article provides an outline of relevant up-to-date knowledge about butyric acid, and presents the expert position on the clinical benefits of using butyric acid products in the therapy of gastrointestinal diseases.
\end{abstract}

\section{Introduction}

The properties of butyric acid, and the role it plays in the gastrointestinal tract, have been known for a long time now. However, butyric acid is still subject to intensive research which shows that it is a factor in the pathogenesis of gastrointestinal diseases, and has a range of previously unknown properties and potential therapeutic applications. Butyric acid remains a molecule with a potential that has not as yet been fully elucidated and realized.

The article provides an outline of relevant up-todate knowledge about butyric acid, and presents the position of gastroenterology and surgery experts on the clinical benefits of using butyric acid products in the therapy of patients with gastrointestinal diseases. Since the article is a follow-up to the study published in 2010 [1], a number of issues already addressed in detail in the previous publication are left out, including the formation and roles of butyric acid in the gastrointestinal tract, problems associated with its deficiency, clinical indications and basic use recommendations.

\section{Immunomodulatory activity of butyric acid in the gastrointestinal tract}

Research conducted in recent years provides evidence for the long postulated mechanism of action of butyric acid, i.e. its effect on the gastrointestinal immune system. In studies on mice, Furusawa et al. [2] showed that the concentration of butyric acid on the colonic wall stimulates the differentiation of $\mathrm{T}_{\text {reg }}$ lymphocytes, reducing the severity of inflammation induced by the transfer of $\mathrm{TCD}^{+}{ }^{+} \mathrm{CD} 45 \mathrm{RB}^{\text {hi }}$ lymphocytes into $\mathrm{Rag}^{1-/}$ mice. Furthermore, in an in vitro study on undifferentiated $T$ cells incubated with butyric acid, the authors demonstrated an increased acetylation of histone $\mathrm{H} 3$, which points to a possible mechanism by which butyric acid (produced by bacteria residing in the gastrointestinal tract) affects the differentiation and specialization of $T_{\text {reg }}$ lymphocytes. These very interesting studies have shed new light on the relationship between the host and the gut microflora, and its impact on immune homeostasis in the gut.

Gut-associated lymphoid tissue (GALT) and enterocytes (intestinal epithelial cells - IECS) act as the first barrier of defence against bacterial invasion through the secretion of mucins and/or defensins (antibacterial peptides) or the detection of pathogens by Toll-like receptors (TLRs). In addition, specialized IECs are capable of transporting bacterial antigens and presenting them to the immune cells in the lamina propria of the gut wall. The system becomes disturbed, for example, in the elderly, as the relationships and proportions between 
bacterial groups forming the microbiome are thrown off balance [3]. Similar unfavourable alterations in the microbiome composition are observed in inflammatory bowel diseases (IBD) or in irritable bowel syndrome (IBS), which is described in subsequent sections below. For many years butyric acid has been postulated to play a role of a primary messenger between commensal bacteria and the human immune system. In this understanding, the presence of butyric acid at an appropriate physiological concentration in the colonic lumen is interpreted by the body as information that the bacterial equilibrium is not disturbed, which reduces immune response and tolerance to thousands of antigens representing commensal bacteria and, hence, prevents the initiation of the inflammatory process [4].

\section{Role of butyric acid in irritable bowel syndrome}

Symptoms associated with gastrointestinal dysfunction, such as IBS, are estimated to occur in 10-30\% of the population. The main signs of the disorder include abdominal pain, diarrhoea and altered conditions in the colon. Patients are typically advised to adopt dietary and lifestyle modifications, and are referred for psychotherapy. Pharmacological management includes antibiotics which act topically in the gastrointestinal lumen (rifaxamin) and, additionally, probiotics and in some cases antidepressants.

In one study, the microbiomes of 113 patients with IBS and 66 control subjects were analyzed. A statistically significant decrease in the amount of butyric acid-producing bacteria was found in the group of patients with IBS, particularly with IBS-D and IBS-M $(p=0.002)$. Also, there was a statistically significant reduction in the level of methane-producing bacteria (Methanobacteria) $(p=0.005)$, which increases local oxygen reservoirs and probably contributes to an increased incidence of flatulence in this patient group [5].

Considering the above, a compound which offers a high chance for success in the therapy of dysfunctional bowel disorders is sodium butyrate. In 2012 Tarnowski et al. [6] assessed the effect of sodium butyrate on selected clinical parameters in patients with irritable bowel syndrome during a 6-week follow-up. The patients were divided into two groups: control group (29 patients) receiving standard treatment with trimebutine and mebeverine (depending on the characteristics of the disease) throughout the entire follow-up period, and study group receiving sodium butyrate at $300 \mathrm{mg}$ daily as an add-on to standard therapy. At baseline and 6 weeks into the study, a questionnaire was completed to assess the symptoms of the disease (on a scale from 0 to 5 ) and the quality of life IBS-QoL (on a scale from 0 to 100). At the time of inclusion in the study, there were no differences between the patient groups in the severity of discomfort and pain, bowel movement disorders, severity of flatulence and other gastrointestinal and IBS-associated symptoms. After 6 weeks, a statistically significant improvement was observed in the study group for the symptoms listed above. What is more, a significant improvement was achieved in the subjective assessment of the quality of life in all patients receiving sodium butyrate.

In 2012 Banasiewicz et al. [7] conducted a randomized clinical trial in a group of 66 patients with a long history of IBS diagnosed on the basis of the ROME II Diagnostic Criteria. The patients were divided into the control group $(n=32)$ and the study group receiving sodium butyrate $(n=34)$ at $300 \mathrm{mg} /$ day $(2 \times 150 \mathrm{mg})$. The follow-up was 4 , and then 12 weeks, with data collected using the Visual Analogue Scale for IBS (VAS-IBS). The following symptoms were assessed: pain in the epigastric region, incidence of flatulence, bowel movement disorders, mucus in stool and the quality of life (based on the IBS-QoL questionnaire). Four weeks after the start of the study, a significant decrease in the incidence of epigastric pain and a reduction in the severity of pain after meals were noted in the group receiving sodium butyrate. After 12 weeks, there was a statistically significant decrease in the incidence of all the symptoms studied, accompanied by an improvement in the patients' QoL. In subsequently published results [8], based on the closed question (yes or no) "Did you achieve good relief from abdominal discomfort or pain associated with IBS during the last week preceding the follow-up visit?", "Yes" answers were achieved respectively in 32 and $6.25 \%$ of the patients $(p<0.01)$ in the study and control groups at week 4 of the study, and in 53 and $15.6 \%$ of the patients $(p<0.01)$, respectively, at week 12 of the study. Importantly, patients in both groups continued previously prescribed therapy (e.g. drotaverine, trimebutine) throughout the whole duration of the study, and received standard treatment for at least 3 months before the inclusion in the study. The authors concluded that butyric acid used as adjunct therapy in the treatment of IBS reduced the incidence of selected clinical symptoms, however without any effect on their severity.

Abdominal pain in patients with IBS is typically a result of disorders related to digestion and fermentation, and the build-up of gases in the gut lumen. The underlying causes of functional diseases have not been fully investigated and require further study, however existing hypotheses suggest that pain is a consequence of transmission abnormalities in the gut-brain axis. In patients with intestinal dysfunctions (IBS) sodium bu- 
tyrate is one of the key factors contributing to gut homeostasis, enhancing natural processes of healing and regeneration in the intestinal epithelium. A decreased incidence of pain characteristic of IBS may be attributable to a diminished receptor sensitivity in the gut [9]. Based on an animal model butyric acid was shown to have an ability to increase the neuronal concentration in the Enteric Nervous System via phenotypic changes in the enteric neurons [10], which in turn has a favourable effect on colonic transit [11].

Another study assessed the efficacy of butyric acid in IBS. Fifty patients with IBS were divided into two subgroups - IBS with constipation (IBS-C) and IBS with diarrhoea (IBS-D) - and treated with butyric acid in the form of enteric-coated tablets at a dose of $1 \mathrm{~g} /$ day. The IBS form and the severity of symptoms were recorded at baseline and at the end of the study. Butyric acid treatment resulted in a reduction or normalization of symptoms in $71 \%$ of patients with IBS-D and in $16 \%$ of patients with IBS-C $(p<0.005)$ [12].

As of today, more than 1,000 bacteria residing in the human gastrointestinal tract have been detected. A vast majority of them belong to about a dozen genera. In adults, Firmicutes and Bacteroidetes are the most abundant phyla, and the Firmicutes to Bacteroidetes ratio appears to determine the bacterial balance in the gastrointestinal tract (in healthy adults Firmicutes is the dominant phylum, however the proportion evolves with age and changes as a result of specific pathological processes) [13]. Pozuelo et al. [5] studied the microbiome in 113 patients with IBS and 66 healthy individuals. Stool samples were collected from the study's subjects twice, at a monthly interval. Bacterial diversity in patients with IBS was found to be reduced in a statistically significant manner, which resulted from a considerably lower abundance of butyrogenic bacteria ( $p=0.002$, $q<0.06$ ), particularly in patients with IBS-D and mixed IBS. The findings of the study are important for considering the benefits of using probiotics in patients with IBS. The most popular probiotic products contain lactic acid bacteria. Assuming colonic colonization with these strains, an increase in local lactate production can be achieved. Tsukahara et al. identified among the commensal bacteria residing in the human gastrointestinal tract a group of Megasphaera elsdenii bacteria belonging to Firmicutes which have an ability to convert lactates into butyrate [14]. Perhaps the same mechanism is responsible for the observed efficacy of lactic acid bacteria in IBS.

\section{Functional constipation}

The composition of bacterial flora is determined by two main classes of bacteria: Firmicutes (a phylum of common Gram-positive bacteria comprising, among others, Clostridium, lactic bacteria and butyric-acid producing bacteria) and Bacteroidetes (Gram-negative rods classified as obligate anaerobes), making up 90-99\% of the total microbiome; Firmicutes is the dominant phylum (50-80\% of the microbiome) [15].

A study conducted in a group of 161 individuals aged 65-96 years showed that their stool microbiota composition (assessed by molecular biology methods), compared to that determined in nine young volunteers (28-46 years old), shifted significantly toward Bacteroidetes [16].

Basic research in an animal model demonstrated that butyric acid increased the effectiveness of peristalsis by improving colonic smooth muscle contractility and regulating neurotransmission, particularly in cases of impaired peristalsis accompanying functional constipation in the elderly [17].

A double-blind, randomized, placebo-controlled study conducted in a group of 11 healthy volunteers involved self-administration of enemas by the study's subjects according to the following regimen: enema with $100 \mathrm{mmol} / /$ of butyric acid in week 1; $50 \mathrm{mmol} / \mathrm{l}$ of butyric acid in week 2; and placebo (saline solution) in week 3 of the study. At the start and end of each test period, a rectal barostat measurement was performed to determine the severity of pain, discomfort, and the urge to pass gas or stool. Butyric acid administered at $50 \mathrm{mmol} / \mathrm{l}$ resulted in a decrease in pain score by $23.9 \%$, at $100 \mathrm{mmol} / \mathrm{l}$ - by $42.1 \%$; and a decrease in discomfort score by $44.2 \%$ and $69.0 \%$, respectively, at a pressure of $4 \mathrm{~mm} \mathrm{Hg}$. The authors concluded that rectal administration of butyric acid led to a dose-dependent decrease in visceral sensitivity which plays a key role in disorders of intestinal motility (including functional constipation), abdominal pain or discomfort [18].

A beneficial effect of butyric acid as one constituent of a multifaceted mechanism modulating gastrointestinal function has also been stressed in patients with stoma and coexisting constipation. Butyric acid supplementation combined with the use of probiotics should be adopted as one of the basic therapeutic strategies in this patient group, preceding treatment with laxatives [19].

Sodium butyrate has also been introduced into the algorithm of dietary treatment in patients with stoma [20].

\section{Diarrhoea}

Sodium butyrate may also prevent diarrhoea through an increased passive absorption of water in the colon and its effects on the gut microflora [21].

Butyrate is easy to administer and counteracts acute dehydration; it may be used in long-term treatment and prevention of traveller's diarrhoea affecting 
individuals moving from countries with higher hygienic standards to destinations with a lower hygienic status. Even though antibacterial substances bring rapid and tangible therapeutic benefits in the treatment of traveller's diarrhoea, there is no possibility to prevent the illness. In addition to standard recommendations such as additional caution about hygiene and food, travellers are advised to eat warm foods, drink bottled water, and eat only fruit and vegetables with intact skin. Pharmacological recommendations include rifaxamin, fluoroquinolone and Lactobacillus bacteria. Krokowicz et al. [22] made an attempt to demonstrate the preventive activity of a mixture of organic acids in a lipid matrix (250 mg of sodium butyrate; $100 \mathrm{mg}$ of fumaric acid; $60 \mathrm{mg}$ of citric acid; $50 \mathrm{mg}$ of sorbic acid; $40 \mathrm{mg}$ of malic acid) in traveller's diarrhoea. A total of 42 patients completed the study, including 20 in the placebo group and 22 individuals taking the acid mixture 3 days before the journey. The incidence of traveller's diarrhoea was $4.5 \%$ in the treatment group, and $40 \%$ in the placebo group. In addition, a statistically significant improvement was noted in the study group in terms of reduction in the number of bowel movements a day and relief of gastric symptoms (abdominal pain, nausea). Similar beneficial effects are observed after using a mixture of sodium butyrate and silicon dioxide (A300) [23].

Persistent and difficult-to-treat diarrhoea is one of the more common side effects of chemo- and radiotherapy in cancer treatment [24]. Karakulska-Prystupiuk [25] described the case of a patient with diarrhoea which developed during the period of myelosuppression in chemotherapy administered for anaplastic lymphoma. The differential diagnosis included recurrence of the primary disease and gastrointestinal tract infection. Subsequent microbiological tests excluded bacterial and fungal diarrhoea, and cytomegalovirus infection. Diarrhoea caused a considerable weight loss: 12 kg over 2 months. Dietary supplementation with butyric acid at $300 \mathrm{mg}$ daily was initiated. After just several days the patient reported a substantial relief of symptoms and elimination of diarrhoea. In addition, the patient reported an elevated appetite and an increased frequency of meals. During successive follow-up examinations the patient was found to gradually return to his normal body weight.

\section{Non-specific inflammatory bowel diseases}

Butyric acid shows a protective effect in inflammatory response secondary to inflammatory bowel diseases. Recent research indicates that chronic stimulation by interferon $\gamma($ IFN- $\gamma$ ) plays an important role in the formation of inflammation-associated colon cancer, and the development of colitis ulcerosa is linked to the gene encoding
IFN- $\gamma$. An in vitro study conducted on human intestinal epithelial cells sampled from the colon of patients with colitis ulcerosa showed an increased infiltration with unspecified $T$ cells in the mechanism of activation of the signal transducer and activator of transcription 1 (STAT1). Butyric acid was also demonstrated to effectively inhibit STAT1 activation by reducing IFN- $\gamma$ production, which results in the apoptosis of T cells of unspecified type and the suppression of the inflammation [26].

Molecular biological methods offer a possibility to gradually identify the relationship between the microbiome, diet, immune response and, consequently, the development of inflammatory bowel diseases. Experience shows that bowel inflammation may be induced by fat-rich diet. In one study, the composition of the microbiota in intestinal biopsies and stool samples was assessed using gene sequencing methods in a group of 231 subjects. One of the findings was a decrease in the amount of Anaerostipes bacteria belonging to Furmicutes in IBD patients who are active smokers or have a history of smoking. Similarly to Megasphaera elsdenii discussed above, Anaerostipes are bacteria responsible for the conversion of lactate to butyrate. In addition, the study revealed that patients with IBD had a reduced amount of Roseburia and Phascolarctobacterium bacteria which produce butyric and propionic acids in the colon [27]. The described ability of some bacteria to convert lactates to butyric acid may also be effectively induced by the commercially available mixture of eight lactic acid-producing probiotic bacteria - VSL\#3 (Lactobacillus plantarum, Lactobacillus delbrueckii subsp. Bulgaricus, Lactobacillus paracasei, Lactobacillus acidophilus, Bifidobacterium breve, Bifidobacterium longum, Bifidobacterium infantis and Streptococcus salivarius). Studies in humans who used VSL\#3 orally showed the preparation to reduce the clinical symptoms and severity of the inflammatory process in patients with pouchitis (after pancolectomy due to colitis ulcerosa) [28], and mild and moderate colitis ulcerosa [29].

A beneficial effect of sodium butyrate in post-proctocolectomy patients has also been demonstrated in research undertaken by the authors of the present publication. In a group of patients with pouchitis, sodium butyrate was found to be a beneficial element of combined therapy, accelerating the remission of symptoms - primarily diarrhoea and pain. However, the main effect linked to sodium butyrate was a reduction in the incidence and severity of inflammation in patients taking microencapsulated sodium butyrate at $2 \times 200 \mathrm{mg}$ [30].

A high concentration of IgA-coated bacteria plays a role in inducing inflammatory bowel diseases, mainly Crohn's disease. A group of researchers [31] assessed the effect of sodium butyrate on the composition of 
the microbiota in IBD-prone IL-10-/- mice. At 8 weeks old, the mice were divided into three groups (four per group): normal (C57BL/6 - negative control), IL-10-/(positive control) and IL-10 $10^{-/}$treated with sodium butyrate administered in drinking water (study group). The severity of colitis symptoms, and the concentrations of proinflammatory cytokines and short-chain fatty acids were assessed in the proximal section of the colon, whereas the percentage of IgA-coated bacteria and the microbiota composition in stool samples were evaluated by $16 \mathrm{~S}$ ribosomal RNA analysis 4 weeks after the initiation of treatment. The study found that sodium butyrate reduced the histologically observed severity of colitis and decreased the level of tumor necrosis factor $\alpha$ (TNF- $\alpha$ ) and IL-6 in IL-10 $10^{-/}$mice treated with sodium butyrate compared with the positive control. At the level of microbiota composition, a reduction in IgA-coated and Bacteroidetes bacteria, and an increase in the group of Firmicutes, were noted in $\mathrm{IL}-10^{-/-}$mice treated with sodium butyrate. The authors concluded that sodium butyrate lowered the risk of colitis, possibly by modifying the composition of the microbiota, i.e. enriching its biodiversity and reducing the amount of colitogenic IgA-coated bacteria.

\section{Effect in cancer prevention and treatment}

A study by Zhang et al. [32] analyzing human colorectal cancer cell lines (HCT-116 and HT-29) treated with sodium butyrate at concentrations ranging from 0.5-5 $\mathrm{mM}$ found that sodium butyrate inhibited the growth of the studied cancer cells, stimulated autophagy and induced apoptotic cell death, thus revealing a new possible mechanism underlying the anticancer activity of butyric acid.

Mouse studies revealed a $75 \%$ reduction in the risk of colon cancer in animals fed fibre-rich diets in a mechanism with butyric acid-producing colonic bacteria acting as intermediaries - compared to bacteria-free mice. It is concluded that the presence of bacteria producing butyric acid is a prerequisite for a fibre-rich diet to exert its beneficial effect which has been extensively described in the literature [33].

Another interesting role of sodium butyrate was described by Bueno-Carrazco et al. [34]. The authors found that oral administration of sodium butyrate supports the anti-cancer efficacy of photodynamic therapy in astrocytoma cells, most likely in a mechanism based on the modulation of gene expression and differentiation of cancer cells.

The synergistic cytotoxic effect on cancer cells was also demonstrated in a study conducted by Encarnacao et al. [35]. The authors showed that butyric acid increased the sensitivity of resistant cancer cells to irinotecan, a second-line drug used in the treatment of colon cancer. The finding may put a new perspective on the application of irinotecan which is regarded by clinicians who balance benefit against risk in the choice of therapy as a drug associated with uncertainty and interindividual variability of response. In vitro observations of colon cancer cell lines conducted over a period of up to $96 \mathrm{~h}$ showed a significant inhibition of cancer cell proliferation in the group where the cells were simultaneously exposed to irinotecan and butyric acid, compared to the group where only irinotecan was used.

The same group of researchers performed an in vitro assessment of the effect of butyric acid on the uptake of the 18F-labelled glucose analogue (18F-FDG) and the increase in glycolysis in a colon cancer cell line [35, 36]. The results show that the addition of butyric acid reduces the uptake of 18F-FDG and may affect the Warburg effect which is correlated with tumour aggressiveness. The greatest differences were observed at the lowest labelled glucose concentrations which, in turn, most accurately reflects the clinical situation. Furthermore, the results of the study suggest that butyric acid may play a role in cancer cells at an advanced stage of development.

\section{Other applications of butyric acid}

In a randomized prospective clinical trial patients with clinically diagnosed diverticulosis $(n=73)$ were assigned to the control and study groups. The study group received sodium butyrate at $300 \mathrm{mg} /$ day $(2 \times 150 \mathrm{mg})$ with a follow-up examination after 12 months. The study was completed by 30 patients receiving sodium butyrate and 22 control group patients receiving placebo. Patients in the study group declared a decrease in the frequency of clinical symptoms of diverticulosis and a significant reduction in the sensation of abdominal discomfort and pain compared to the placebo group [37].

Sodium butyrate in the form of enemas (combined in a mixture with A-300 silicon dioxide) may be a successful method of therapeutic management in patients with radiation proctitis. Sodium butyrate was shown to have an ability to reduce inflammation, as confirmed by clinical and endoscopic assessment. A key aspect related to sodium butyrate which appears to be of great relevance for this challenging and treatment-resistant form of proctitis is a multifaceted mechanism of action of butyrate which prevents inflammation, stimulates proliferation and normalizes the profile of secreted mucus [38].

\section{Conclusions}

The body of knowledge about the roles and importance of butyric acid has been expanding steadily, and 
the mechanisms by which butyric acid affects the relationship between the microbiome and the host are becoming increasingly elucidated. It is currently believed that the clinical aspects of using butyric acid described above arise from the effect of the compound on the local immune system, the mechanisms regulating the gut peristalsis, the severity of inflammatory processes and the regulatory mechanisms of the gut-brain axis [39]. The complex mechanism of action of butyric acid seems to play a vital role in maintaining symbiosis and homeostasis in the human body.

The scope of the present article is limited to oral butyric acid preparations used at doses which provide no possibility for significant quantities of butyric acid to pass into the systemic circulation. Consequently, it excludes studies focused on the role of butyric acid in reducing peripheral insulin resistance in diabetes mellitus type 2 [40] or preventing body weight gain [41], which represent important future challenges for researches. The coming years are bound to bring new discoveries and fascinating reports on other areas of activity of butyric acid, and therefore new therapeutic opportunities associated with its use.

\section{Conflict of interest}

The authors declare no conflict of interest.

\section{References}

1. Banasiewicz T, Borycka-Kiciak K, Dobrowolska-Zachwieja A i wsp. Kliniczne aspekty zastosowania kwasu masłowego w postępowaniu dietetycznym w chorobach jelit. Prz Gastroenterol 2010; 5: 329-34.

2. Furusawa Y, Obata Y, Fukuda S, et al. Commensal microbe-derived butyrate induces the differentiation of colonic regulatory T cells. Nature 2013; 504: 446-50.

3. Margone T, Jirillo E. The interaction between gut microbiota and age-related changes in immune function and inflammation. Immun Ageing 2013; 10: 31.

4. Louis P, O'Byrne CP. Life in the gut: microbial responses to stress in the gastrointestinal tract. Sci Prog 2010; 93: 7-36.

5. Pozuelo M, Panda S, Santiago A, et al. Reduction of butyrateand methane-producing microorganisms in patients with irritable bowel syndrome. Sci Rep 2015; 5: 12693.

6. Tarnowski W, Borycka-Kiciak K, Kiciak A i wsp. Wyniki leczenia zespołu jelita nadwrażliwego z zastosowaniem kwasu masłowego - raport wstępny. Gastroenterol Prakt 2011; 1: 43-8.

7. Banasiewicz T, Kaczmarek E. Wpływ chronionego maślanu sodu na częstość i nasilenie wybranych objawów klinicznych $u$ chorych z zespołem jelita nadwrażliwego. Gastroenterol Prakt 2012; 1: 48-54.

8. Banasiewicz T, Krokowicz Ł, Stojcev Z, et al. Microencapsulated sodium butyrate reduces the frequency of abdominal pain in patients with irritable bowel syndrome. Colorectal Dis 2012; 15: 204-9.
9. Garland EL, Gaylord SA, Palsson O, et al. Therapeutic mechanisms of a mindfulness-based treatment of IBS: effect of visceral sensitivity, catastrophizing, and affective processing of pain sensations. J Behav Med 2011; 35: 591-602.

10. Soret R, Chevalier J, De Coppet P, et al. Short-chain fatty acids regulate the enteric neurons and control gastrointestinal motility in rats. Gastroenterology 2010; 138: 1772-82.

11. de Giorgio R, Blandizzi C. Targeting enteric neuroplasticity: diet and bugs as new key factors. Gastroenterology 2010; 138: 1663-6.

12. Scarpellini E, Lauritano EC, Lupascu A, et al. Efficacy of butyrate in the treatment of diarrhoea-predominant irritable bowel syndrome. Dig Liver Dis 2007; Suppl 1: 19-22.

13. Tojo R, Suárez A, Clemente MG, et al. Intestinal microbiota in health and disease: role of bifidobacteria in gut homeostasis. World J Gastroenterol 2014; 20: 15163-76.

14. Tsukahara T, Koyama H, Okada M, Ushida K. Stimulation of butyrate production by gluconic acid in batch culture of pig cecal digesta and identification of butyrate-producing bacteria. J Nutr 2002; 132: 2229-34.

15. Biagi E, Candela M, Fairweather $S$, et al. Ageing of the human metaorganism: the microbial counterpart. AGE 2012; 34: 247-67.

16. Claesson MJ, Cusack S, O'Sullivan O, et al. Composition, variability, and temporal stability of the intestinal microbiota of the elderly. Proc Natl Acad Sci U S A 2011; 108 Suppl. 1: 4586-91.

17. Bajka BH, Clarke JM, Topping DL, et al. Butyrylated starch increases large bowel butyrate levels and lowers colonic smooth muscle contractility in rats. Nut Res 2010; 30: 427-34.

18. Vanhoutvin SA, Troost FJ, Kilkens TO, et al. The effects of butyrate enemas on visceral perception in healthy volunteers. Neurogastroenterol Motil 2009; 9: 952e76.

19. Kuczynska B, Bobkiewicz A, Studniarek A, et al. Conservative measures for managing constipation in patients living with a colostomy. J Wound Ostomy Continence Nurs 2017; 44: 160-4.

20. Krokowicz L, Bobkiewicz A, Borejsza-Wysocki M, et al. A prospective, descriptive study to assess the effectiveness of dietary and pharmacological strategies to manage constipation in patients with a stoma. Ostomy Wound Manage 2015; 61: 14-22.

21. Velázquez OC, Ledered HM, Rombeau JL. Butyrate and the colonocyte. Production, absorption, metabolism and therapeutic implications. Adv Exp Biol 1997; 427: 123-34.

22. Krokowicz Ł, Kaczmarek BF, Krokowicz P, et al. Sodium butyrate and short chain fatty acids in prevention of travellers' diarrhoea: a randomized prospective study. Travel Med Infect Dis 2014; 12: 183-8.

23. Krokowicz $九$, Mackiewicz J, Wejman-Matela A, et al. Management of traveller's diarrhoea with a combination of sodium butyrate, organic acids, and A-300 silicon dioxide. Prz Gastroenterol 2014; 9: 285-90.

24. Andreyev HJN, Davidson SE, Gillespie C, et al. Practice guidance on the management of acute and chronic gastrointestinal problems arising as a result of treatment for cancer. Gut 2012; 61: 179-92.

25. Karakulska-Prystupiuk E. Butyric acid in inhibition of diarrhoea in course of chemotherapy - case report. Onkol Prakt Klin 2011; 7: 40-2.

26. Zimmerman MA, Singh N, Martin PM, et al. Butyrate suppresses colonic inflammation through HDAC1-dependent Fas up- 
regulation and Fas-mediated apoptosis of T cells. Am J Physiol Gastrointest Liver Physiol 2012; 302: G1405-15.

27. Morgan XC, Tickle TL, Sokol H, et al. Dysfunction of the intestinal microbiome in inflammatory bowel disease and treatment. Genome Biol 2012; 13: R79.

28. Gionchetti P, Rizzello F, Helwig U, et al. Prophylaxis of pouchitis onset with probiotic therapy: a double-blind, placebo-controlled trial. Gastroenterology 2003; 124: 1202-9.

29. Sood A, Midha V, Makharia GK, et al. The probiotic preparation, VSL\#3 induces remission in patients with mild-to-moderately active ulcerative colitis. Clin Gastroenterol Hepatol 2009; 7: 1202-9.

30. Kuczyńska B, Paszkowski J, Krokowicz Ł i wsp. Chroniony maślan sodu w profilaktyce i leczeniu zapalenia błony śluzowej zbiornika jelitowego (pouchitis). X Jubileuszowe Sympozjum Polskiego Klubu Koloproktologii, 11-13 września 2014, Serock (prezentacja ustna).

31. Zhang T, Ding C, Zhao M, et al. Sodium butyrate reduces colitogenic immunoglobulin A-coated bacteria and modifies the composition of microbiota in IL-10 deficient mice. Nutrients 2016; 8: 728.

32. Zhang J, Yi M, Zha L, et al. Sodium butyrate induces endoplas mic reticulum stress and autophagy in colorectal cells: implications for apoptosis. PLoS One 2016; 11: e0147218.

33. Donohoe DR, Holley D, Collins LB, et al. A gnotobiotic mouse model demonstrates that dietary fiber protects against colorectal tumorigenesis in a microbiota- and butyrate-dependent manner. Cancer Discov 2014; 4: 1387-97.

34. Bueno-Carrazco J, Castro-Leyva V, García-Gomez F, et al. Sodium butyrate increases the effect of the photodynamic therapy: a mechanism that involves modulation of gene expression and differentiation in astrocytoma cells. Child's Nervous System 2012; 28: p1723.

35. Encarnacao JC, Pires AS, Goncalves TJ, et al. Butyrate and irinotecan combination as a new therapeutic approach for colon cancer, EACR-23 Poster Sessions. Eur J Cancer 2014; 50 Suppl. 5: S23-242.

36. Encarnacao JC, Pires AS, Goncalves TJ, et al. Is 18F-FDG uptake in three different colon cancer cell lines affected by incubation with sodium butyrate? EACR-23 Poster Sessions. Eur J Cancer 2014; 50 Suppl. 5: S23-242.

37. Krokowicz L, Stojcev Z, Kaczmarek BF, et al. Microencapsulated sodium butyrate administered to patients with diverticulosis decreases incidence of diverticulitis - a prospective randomized study. Int J Colorectal Dis 2014; 29: 387-93.

38. Stojcev Z, Krokowicz L, Krokowicz P, et al. Early treatment and prevention of the radiation proctitis-composite enemas containing sodium butyrate. Int J Colorectal Dis 2013; 28: 1731-2.

39. Marchesi JR, Adams DH, Fava F, et al. The gut microbiota and host health: a new clinical frontier. Gut 2016; 65: 330-9.

40. Khan S, Jena GB. Protective role of sodium butyrate, a HDAC inhibitor on beta-cell proliferation, function and glucose homeostasis through modulation of p38/ERK MAPK and apoptotic pathways: Study in juvenile diabetic rat. Chemico-Biological Interactions 2014; 213: 1-76 (25 April 2014).

41. Lin HV, Frassetto A, Kowalik Jr EJ, et al. Butyrate and propionate protect against diet-induced obesity and regulate gut hormones via free fatty acid receptor 3-independent mechanisms. PLoS One 2012; 7: e35240.
Received: 8.05.2017

Accepted: 9.06.2017 